\title{
ESTUDO DA REAÇÃO DE OXIDAÇÃO SELETIVA DE ALDEIDOS INSATURADOS. ETAPA I: PREPARAÇÃO DE CATALISADORES
}

\author{
W. M. FANTIM ${ }^{1}$, L. H. VIEIRA ${ }^{2}$, L. MARTINS ${ }^{2}$, R. CONDOTTA ${ }^{1}$, J. G. R. POCO ${ }^{1}$ \\ ${ }^{1}$ Centro Universitário da FEI, Departamento de Engenharia Química \\ ${ }^{2}$ Universidade Estadual de São Paulo, Araraquara, Departamento de Engenharia Química \\ E-mail para contato: wellina_MF@hotmail.com
}

\begin{abstract}
RESUMO - O ácido acrílico é muito utilizado na fabricação de polímeros superabsorventes e é obtido a partir da oxidação do propeno, proveniente de fontes petroquímicas. $\mathrm{O}$ processo ocorre por meio de duas reações com condições e catalisadores diferentes. A primeira reação é a para a obtenção de acroleína utilizando catalisadores à base de molibdênio, bismuto e promotores e a segunda reação é a oxidação de acroleína a ácido acrílico, que ocorre na presença de catalisadores de molibdênio, vanádio e promotores. Neste trabalho foram realizadas diversas preparações para este segundo tipo de catalisador seguindo procedimentos descritos em patentes de domínio público. Os catalisadores foram caracterizados por infravermelho (FTIR), difração e fluorescência de raio-X, para observação do efeito da temperatura de calcinação na amostra. A seguir, os catalisadores foram testados em reator utilizando a glicerina, fonte renovável alternativa ao uso do propeno, como reagente, fornecendo rendimentos de até $28 \%$ para o ácido acrílico.
\end{abstract}

\section{INTRODUÇÃO}

\subsection{Reações Catalíticas}

Catalisadores são substâncias que facilitam a conversão dos reagentes em produtos, não participam do balanço material da reação e são regenerados ao seu estado inicial ao final de cada ciclo, interferindo apenas na cinética da reação e não em sua termodinâmica. Podem ser homogêneos, ou heterogêneos, sendo que os últimos são mais utilizados por serem mais fáceis de separá-los da solução, já que geralmente são sólidos enquanto os reagentes são líquidos ou gasosos. (DUMESIC, 2008).

Um bom catalisador é aquele que possui alta seletividade ao produto desejado e baixa para outros subprodutos, deve ter um desempenho estável por longos períodos de tempo e principalmente uma boa atividade catalítica que é característica das interações entre sua superfície catalítica e os reagentes (DUMESIC, 2008).

O processo para preparação de catalisadores ocorre em duas etapas, a primeira envolve os fenômenos químicos e a segunda os tratamentos térmicos que se traduzem por fenômenos 
preponderantemente físicos. Os catalisadores mais utilizados são formados por uma fase do material ativo e a outra do suporte, cuja função é dispersar essa área ativa.

\subsection{Processo para obtenção do ácido acrílico}

O ácido acrílico é obtido por meio da oxidação do propeno, que acontece em duas reações sequenciais, com catalisadores e condições distintas, as quais podem se otimizadas separadamente. Para a oxidação seletiva do propeno são utilizados catalisadores contendo principalmente molibdênio e bismuto suportados em sílica, que oferecem rendimentos maiores que $90 \%$. Já para a oxidação da acroleína os catalisadores contêm principalmente molibdênio e vanádio, numa razão atômica elevada. (GRASSELLI, 2008).

$$
\begin{array}{ll}
\mathrm{C}_{3} \mathrm{H}_{6}+\mathrm{O}_{2} \stackrel{\text { Cat. } \mathrm{A}}{\longrightarrow} \mathrm{C}_{3} \mathrm{H}_{4} \mathrm{O}+\mathrm{H}_{2} \mathrm{O} & \Delta \mathrm{H}=-340,8 \mathrm{~kJ} / \mathrm{mol} \\
\mathrm{C}_{3} \mathrm{H}_{4} \mathrm{O}+1 / 2 \mathrm{O}_{2} \stackrel{\text { Cat. } \mathrm{B}}{\longrightarrow} \mathrm{C}_{3} \mathrm{H}_{4} \mathrm{O}_{2} & \Delta \mathrm{H}=-254,1 \mathrm{~kJ} / \mathrm{mol}
\end{array}
$$

\section{PROCEDIMENTO}

Os catalisadores para a oxidação de acroleína a serem utilizados na oxidação de glicerina foram preparados baseando-se em procedimentos descritos em patentes que estão em domínio público, citadas na Tabela 1.

Tabela 1: Composição atômica metálica dos catalisadores preparados e suas patentes

\begin{tabular}{|c|c|}
\hline Catalisador & Referência \\
\hline $\mathrm{Mo}_{12} \mathrm{~V}_{1.9} \mathrm{Si}_{10} \mathrm{Cu}_{2.2}$ & US 3567772 (, (Yanagita, 1971) \\
\hline $\mathrm{Mo}_{12} \mathrm{~V}_{3} \mathrm{~W}_{0,5} \mathrm{O}_{42,5}$ & US 3567773, (Yamaguchi, 1971) \\
\hline $\mathrm{Mo}_{12} \mathrm{~V}_{3} \mathrm{~W}_{1,2} \mathrm{Cu}_{1} \mathrm{Sb}_{6}$ & US 3773692, (Hensel, 1973) \\
\hline $\mathrm{Mo}_{12} \mathrm{~V}_{4.8} \mathrm{~W}_{2.4} \mathrm{Cu}_{2.2}$ & US3954855, (Wada, 1976) \\
\hline $\mathrm{Mo}_{12} \mathrm{~V}_{1} \mathrm{Si}_{3}$ & US 3773828 (Kadowaki, 1973) \\
\hline $\mathrm{Mo}_{12} \mathrm{~V}_{3} \mathrm{~W}_{1,2} \mathrm{Ce}_{3}$ & GB 1477029 Sohio (The Standard Oil, 1973) \\
\hline $\mathrm{Mo}_{12} \mathrm{~V}_{3} \mathrm{~W}_{1,2} \mathrm{Sb}_{3} \mathrm{Sn}_{3}$ & US 3840595 Sohio (Grassselli, 1974) \\
\hline $\mathrm{Mo}_{12} \mathrm{~V}_{4.7} \mathrm{~W}_{1,1} \mathrm{Cu}_{6,3}$ & US 3997600 Societá Italiana (Ferlazzo, 1976) \\
\hline
\end{tabular}

Em geral, os catalisadores foram preparados a partir de reagentes e formas de calcinação diferentes, conforme descrito na Tabela 2. Durante as preparações, todos os sais fontes dos elementos utilizados foram dissolvidos separadamente em água quente e depois misturados entre si, conforme indicação das patentes, formando uma solução ou mistura principal, na qual foi adicionada uma fonte de sílica (Aerosil® ou Ludox®) sob agitação. Em seguida, a solução foi elevada à temperatura de $80{ }^{\circ} \mathrm{C}$ até evaporação total do solvente, sob agitação a 500 rpm com o auxílio de um agitador mecânico durante cerca de 2 horas. 
Tabela 2: Reagentes utilizados durante as preparações e condições para a calcinação

\begin{tabular}{|c|c|c|c|c|c|c|c|c|}
\hline Reagentes & 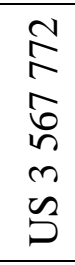 & $\begin{array}{l}n \\
n \\
\infty \\
\dot{v} \\
2 \\
m \\
\infty \\
b\end{array}$ & 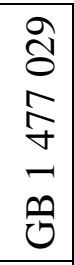 & $\begin{array}{l}8 \\
8 \\
\hat{\sigma} \\
\text { n } \\
\tilde{b}\end{array}$ & 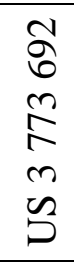 & 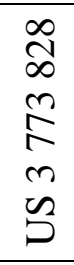 & $\begin{array}{l}n \\
\hat{N} \\
\hat{\delta} \\
n \\
n \\
\tilde{\sigma} \\
n\end{array}$ & 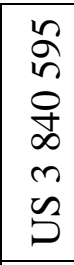 \\
\hline Heptamolibdato de amônio (g) & 9,02 & 14,1 & 7,5 & 13,3 & 17,7 & 19,1 & 21,3 & 10,9 \\
\hline Metavanadato de amônio (g) & 0,94 & 3,61 & 1,3 & 5,02 & 3,02 & 2,72 & 2,35 & 1,77 \\
\hline Nitrato de cobre $(\mathrm{g})$ & 2,09 & 3,62 & - & 11,6 & 2,27 & - & - & - \\
\hline Tungstato de amônio $(\mathrm{g})$ & - & 3,9 & 1 & 4,51 & 2,44 & - & 1,4 & 1,45 \\
\hline Antimoniato de potássio $(\mathrm{g})$ & - & - & - & - & 13,1 & - & - & 3,93 \\
\hline Hidróxido de amônio $(16 \%)(\mathrm{ml})$ & - & - & - & 400 & - & - & - & - \\
\hline Oxido de estanho II (g) & - & - & - & - & - & - & - & 2,25 \\
\hline Ácido Oxálico (g) & & & & & & 8,02 & & \\
\hline Nitrato de cério $(\mathrm{g})$ & & & 3,1 & & & & & \\
\hline Sílica coloidal $30 \%$ (g) & & 41,1 & & & & & 11,8 & 35 \\
\hline Sílica coloidal $40 \%(\mathrm{~g})$ & 38,7 & & & & & & & \\
\hline Sílica pirogênica Aerosil ${ }^{\circledR}(\mathrm{g})$ & & & 15 & & 17,6 & 4,05 & & \\
\hline \multicolumn{9}{|c|}{ Calcinação } \\
\hline Tempo (h) & 6 & 5 & 2 & 2 & 2 & 5 & 5 & 24 \\
\hline Temperatura $\left({ }^{\circ} \mathrm{C}\right)$ & 400 & 400 & 400 & 370 & 450 & 370 & 500 & 130 \\
\hline
\end{tabular}

Após secagem em estufa a $100{ }^{\circ} \mathrm{C}$, as amostras foram calcinadas nas temperaturas e durante os períodos de tempo sugeridos pelas patentes, conforme descrito na Tabela 2. A seguir, foram caracterizadas por infravermelho (FTIR) (diluídas em pastilhas de $\mathrm{KBr}$ ) e difração de raios-X no pó. Posteriormente, os catalisadores tiveram seus desempenhos avaliados em reator na reação de oxi-desidratação da glicerina. As condições utilizadas foram: vazão de $3 \mathrm{~mL} / \mathrm{min}$ de uma corrente contendo $10 \%$ glicerol/água, a uma temperatura de $350{ }^{\circ} \mathrm{C}$ e $0,2 \mathrm{~g}$ de cada catalisador e vazão de uma mistura de $\mathrm{N}_{2}$ e $\mathrm{O}_{2} 80 / 20$ de $30 \mathrm{~mL}$ por minuto.

\section{RESULTADOS E DISCUSSÕES}

Para cada catalisador produzido foram feitas caracterizações por infravermelho e raio-X para todas as amostras calcinadas a diferentes temperaturas, podendo-se verificar as alterações sofridas pela fase cristalina em função da temperatura de calcinação. Nas figuras a seguir estão representados os espectros obtidos na análise dos catalisadores US 3567772 e US 3 954855 , exemplificando o estudo realizado. As mesmas análises foram feitas nos demais catalisadores. 
A Figura 1 apresenta os espectros de infravermelho das amostras calcinadas a diferentes temperaturas segundo a patente US 3567 772. Analisando-se os difratogramas da Figura 2, percebe-se que as amostras B1.1 e B1.3 calcinadas a $400{ }^{\circ} \mathrm{C}$ e $500{ }^{\circ} \mathrm{C}$ apresentam cristalinidade menor já que seus picos são pouco intensos. A amostra calcinada a $400{ }^{\circ} \mathrm{C}$ apresentou picos semelhantes ao $\mathrm{SiO}_{2}, \mathrm{VO}_{2}$ e $\mathrm{MoO}_{3}$. A amostra $\mathrm{B} 1.3$, calcinada a $500{ }^{\circ} \mathrm{C}$ apresentou uma mudança de fase cristalina e teve como principais constituintes o $\mathrm{MoO}_{3}$, $\mathrm{CuMoO}_{4}$ e $\mathrm{VO}_{2}$. Já a amostra calcinada a $600{ }^{\circ} \mathrm{C}$ tem picos semelhantes ao $\mathrm{Cu}_{3} \mathrm{Mo}_{2} \mathrm{O}_{9}$, e manteve o óxido de molibdênio, indicando que não houve perda da fase ativa na calcinação a alta temperatura.

Figura 1: Espectros infravermelhos das amostras US 3567772 .

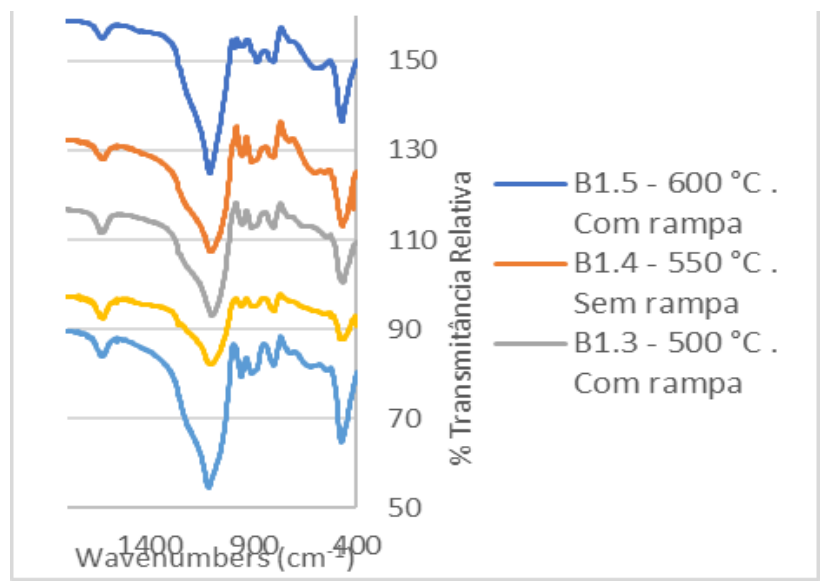

Figura 2: Difrações de raios-X da amostra US 3567772

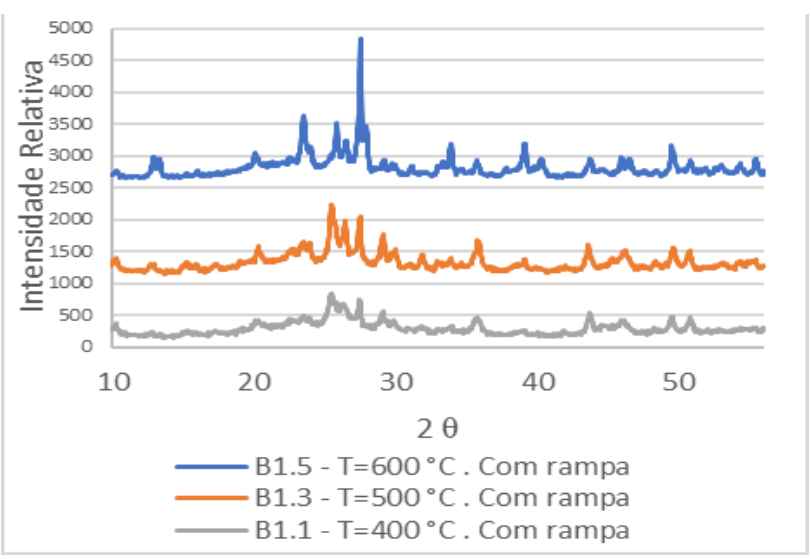

As figuras 3 e 4 apresentam os espectros de infravermelho e difratogramas de raios-X da amostra preparada segundo a patente US 3954 855. Nos difratogramas da amostra B2 é possível notar que a amostra calcinada a $400{ }^{\circ} \mathrm{C}$ praticamente não apresenta picos, assim só foi possível identificar a presença de $\mathrm{VO}_{2}$. A amostra calcinada a $500{ }^{\circ} \mathrm{C}$ apresentou maior cristalinidade e picos semelhantes a substâncias como $\mathrm{MoO}_{3}$ e $\mathrm{CuMoO}_{4}$. Já a amostra calcinada a $600{ }^{\circ} \mathrm{C}$ apresentou picos mais intensos e diferentes dos anteriores, indicando a presença de $\mathrm{MoO} 3, \mathrm{~V}_{4} \mathrm{O}_{7}, \mathrm{Cu}_{3} \mathrm{Mo}_{2} \mathrm{O}_{9}$ e $\mathrm{WO}_{2,92}$. Nesse caso evidenciou-se a ocorrência de mudanças de fase durante a calcinação. 
Figura 3: Espectros infravermelhos das amostras US 3954855.

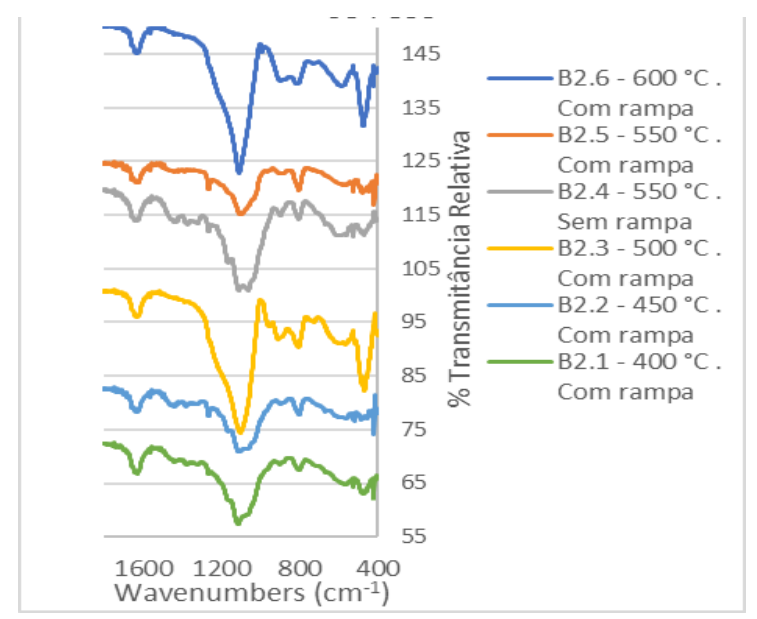

Figura 4: Difrações de raios-X da amostra US 3954855

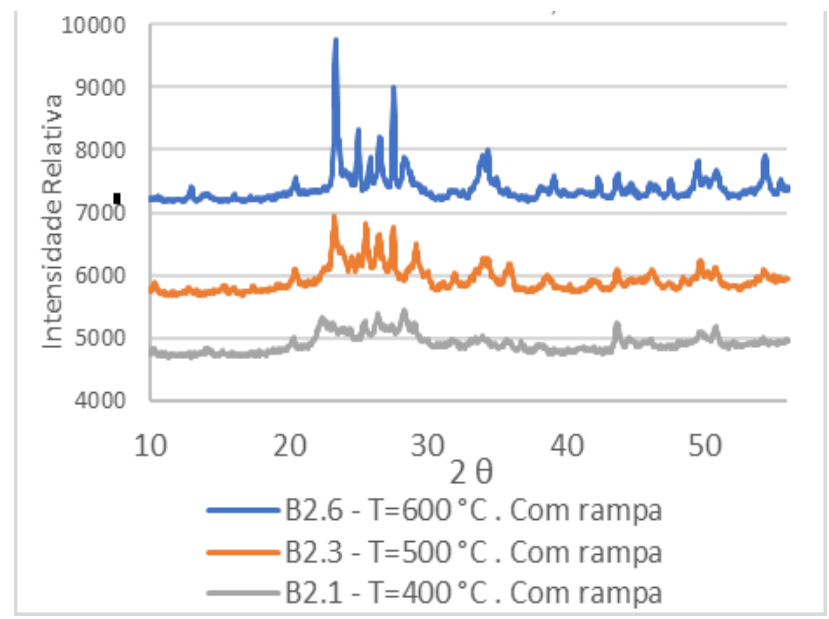

Figura 5: Resultados obtidos durante o teste em reator para os catalisadores para oxidação da acroleína (Tipo B)

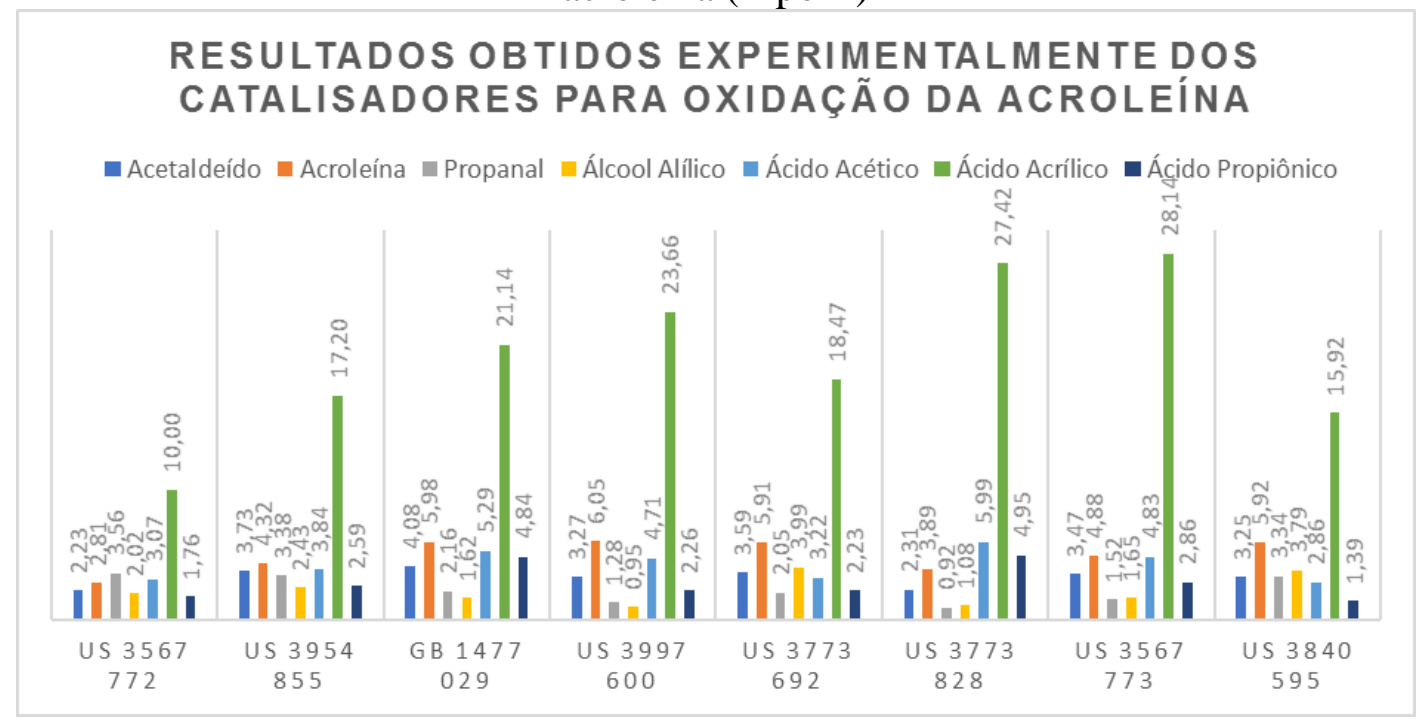




\section{CONCLUSÃO}

Concluiu-se que a forma de preparação de catalisador envolve vários fatores que podem interferir no resultado final, tais como a seleção dos reagentes e a escolha da temperatura de calcinação que pode influenciar tanto na formação de fase ativa, (ex: estrutura cristalina e composição superficial), como na perda de componentes dessa fase ativa por sublimação.

\section{REFERÊNCIAS}

DUMESIC, J. A.; HUBER, G. W.; BOUDART, M. In: ERTL, G. et al. Handbook of Heterogeneous Catalysis ( $2^{\text {nd }}$ Edition) V1 p1-14. Wiley-VCH Verlag GmbH \& Co. KGaA, Weinheim, Germany 2008.

FANTIM, W. M. Estudo Da Reação De Oxidação Seletiva De Aldeídos Insaturados: Etapa I - Preparação De Catalisadores. Relatório Final de Iniciação Cientifica, FEI, São Bernardo do Campo, 2016.

FERlaZZO, N; FAUSTO, G. B.; GHIRGA, M.; Method of Preparing Acrylic Acid; US Patent 3,997,600, 1976

GRASSELLI, R.; FALLS, C.; SURESH, D.; HEIGHTS, W.; Process for the Conversion of Unsaturated Aldehydes to Acids; US Patent 3,840,595, 1974.

GRASSELLI, R.K.; BURRINGTON, J.D In: ERTL, G. et al. Handbook of Heterogeneous Catalysis (2nd Edition) V7 p3479-3489. Wiley-VCH Verlag GmbH \& Co. KGaA, Weinheim, Germany 2008.

HENSEL, J. et alli Catalysts for the Oxidation of Alpha, Beta-Unsaturated Aldehydes to Alpha, Beta-Unsaturated Carboxylic Acids and Process for Their Preparation; US Patent 3,773,692.

KADOWAKI, K.; KOSHIKAWA, T.; Production of Acrylic Acid; Patented US Patent 3,773,828, 1977.

DOLHYJ, S. R.; MILBERGER, E. C.; Catalyst Compositions Especially Useful for Preparation of Unsaturated Acids; GB Patent 1,477,029, 1977.

SCHUH, K.; KLEIST, W.; TROUILLET, V., BEATO, P., JENSEN, A.D.; GRUNWALDT, J.D. Bismuth Molybdate Catalysts Prepared by Mild Hydrothermal Synthesis: Influence of $\mathrm{pH}$ on the Selective Oxidation of Propylene. Catalysts 2015, 5(3), 1554-1573

WADA, M; YANAGISAWA, I; NINOMIYA, M; OHARA, T; Process for Preparing Acrylic Acid. US Patent 3,954,855, 1976.

YAMAGUCHI, G; TAKENAKA, S; Production of Unsaturated Aliphatic Acids; US Patent 3,567,773, 1971.

YANAGITA, M; KITAHARA, M; Process for the Production of Acrylic Acid; Patented US Patent 3,567,772, 1971 